\title{
On Stability of Differential Systems with Noninstantaneous Impulses
}

\author{
Anju Sood $^{1}$ and Sanjay K. Srivastava $^{2}$ \\ ${ }^{1}$ Applied Sciences Department, Punjab Technical University, Kapurthala, Punjab 144601, India \\ ${ }^{2}$ Faculty of Mathematics, Applied Sciences Department, Beant College of Engineering and Technology, Gurdaspur, Punjab 143521, India
}

Correspondence should be addressed to Anju Sood; anjusood36@yahoo.com

Received 5 January 2015; Accepted 7 March 2015

Academic Editor: Sotiris K. Ntouyas

Copyright (c) 2015 A. Sood and S. K. Srivastava. This is an open access article distributed under the Creative Commons Attribution License, which permits unrestricted use, distribution, and reproduction in any medium, provided the original work is properly cited.

A new class of impulsive differential equations with noninstantaneous fixed time impulses is considered. Uniform stability and uniform asymptotic stability of solutions of the system have been established by employing piecewise Lyapunov functions. An example is also given to illustrate the theoretical results.

\section{Introduction}

Differential equations are one of the most frequently used tools for mathematical modeling in engineering and life sciences. Many evolution processes are subjected to short term perturbations caused by external interventions during their evolution. Very often, the duration of these effects is negligible acting instantaneously in the form of impulses. Many modeled phenomena which have a sudden change in states such as population dynamics, biotechnology process, chemistry, engineering, and medicine can be formulated by the following impulsive differential equations:

$$
\begin{array}{cc}
x^{\prime}=f(t, x), & t \neq t_{k}, \\
\Delta x=I_{i}(x), & t=t_{k},
\end{array}
$$

where $i \in N, t \in R_{+}, I_{i}(x)=x\left(t_{i}^{+}\right)-x\left(t_{i}\right), x \in R^{n}, f: R^{+} \times$ $R^{n} \rightarrow R^{n}, I_{i}: R^{n} \rightarrow R^{n}$, and $0=t_{0}<t_{1}<t_{2}<t_{3}<\cdots \infty$.

Let $t_{0} \in R_{+}, x_{0} \in R^{n}$, such that $x\left(t, t_{0}, x_{0}\right)$ is the solution of system (1), satisfying the initial conditions $x\left(t_{0}+\right.$ $\left.0, t_{0}, x_{0}\right)=x_{0}$. Here, the impulsive conditions are the combination of the traditional initial value problem and the short term perturbations whose duration can be negligible in comparison with the duration of such process [1-9].

However, the above short term perturbations could not show the dynamic change of evolution process completely in pharmacotherapy. We know that the introduction of new drugs in the bloodstream and the consequent absorption for the body are gradual and continuous processes. Thus, we have to use a model to describe such an evolution process. In fact, the above situation has fallen in a new impulsive action, which starts at an arbitrary fixed point and keeps active on a finite time interval. To achieve this aim, a new class of semilinear impulsive differential equations with noninstantaneous impulses is introduced by Hernandez and O'Regan [10] in 2013. Then, the results for the existence of solutions of such equations are established by Pierri et al. [11].

Motivated by [10-13], we introduce a new Lyapunov stability concept for the following semilinear differential equation with noninstantaneous impulses:

$$
\begin{aligned}
& x^{\prime}(t)=f(t, x(t)), \quad t \in\left(s_{i}, t_{i+1}\right], i=0,1,2,3, \ldots, m, \\
& x(t)=g_{i}(t, x(t)), \quad t \in\left(t_{i}, s_{i}\right], i=1,2,3, \ldots, m,
\end{aligned}
$$

where $0=t_{0}=s_{0}<t_{1} \leq s_{1} \leq t_{2} \leq \cdots s_{m-1} \leq t_{m} \leq s_{m} \leq$ $t_{m+1}=T$ are prefixed numbers, $f:[0, T] \times R \rightarrow R$ is continuous, and $g_{i}:\left[t_{i}, s_{i}\right] \times R \rightarrow R$ is continuous for all $i=$ $1,2,3, \ldots, m$.

Practically, in system (2), we consider a new model to describe an evolution processs, in which an impulsive action starts at an arbitrary fixed point and keeps active on a finite 
time interval. Assume that we can measure the state of the process at any time to get a function $x(\cdot)$ as a solution of (2).

The novelty of our paper is to consider a new type of impulsive differential equation (2) with noninstantaneous impulses, finding reasonable conditions to establish Lyapunov's uniform stability and asymptotic uniform stability of solutions [14] of system (2).

In Section 2, we present some preliminaries. In Section 3, uniform stability and asymptotic uniform stability of solutions have been established by using piecewise Lyapunov function. The theoretical results have been illustrated by an example in Section 4.

\section{Preliminaries}

Let $R$ denote the set of real numbers, $J=[0, T]$, and let $C(J, R)$ be the Banach space of all continuous functions from $J$ into $R$ with the norm $\|x(t)\|_{C}=\operatorname{Sup}\{|x(t)|: t \in J\}$ for $x \in C(J, R)$. We introduce the Banach space $P C(J, R)=\{x \in J \rightarrow R: x \in$ $\left.C\left(\left(t_{k}, t_{k+1}\right], R\right), k=0,1,2, \ldots, m\right\}$ and $\exists x\left(t_{k}^{-}\right)$and $x\left(t_{k}^{+}\right)$with $x\left(t_{k}^{-}\right)=x\left(t_{k}\right),\|x(t)\|_{P C}=\operatorname{Sup}\{|x(t)|: t \in J\}$. Meanwhile, we set $P C^{1}(J, R)=\left\{x \in P C(J, R): x^{\prime} \in P C(J, R)\right\}$ with $\|x\|_{P C^{1}}=\max \left\{\|x\|_{P C},\left\|x^{\prime}\right\|_{P C}\right\}$. Clearly, $P C^{1}(J, R)$ endowed with the norm $\|\cdot\|_{P C^{1}}$ is also a Banach space.

By virtue of the concept of solutions in [10] and also used in [12], we introduce the following definition.

Definition 1. A function $x \in P C^{1}(J, R)$ is called a classical solution of the problem

$$
\begin{aligned}
& x^{\prime}(t)=f(t, x(t)), \quad t \in\left(s_{i}, t_{i+1}\right], i=0,1,2,3, \ldots, m, \\
& x(t)=g_{i}(t, x(t)), \quad t \in\left(t_{i}, s_{i}\right], i=1,2,3, \ldots, m, \\
& x(0)=x_{0}, \quad x_{0} \in R,
\end{aligned}
$$

if $x$ satisfies

$$
x(0)=x_{0}
$$

$x(t)$

$$
= \begin{cases}g_{i}(t, x(t)), & t \in\left(t_{i}, s_{i}\right], \\ & i=0,1,2,3, \ldots, m, \\ x_{0}+\int_{0}^{t} f(s, x(s)) d s, & t \in\left[0, t_{1}\right], \\ g_{i}\left(s_{i}, x\left(s_{i}\right)\right)+\int_{s_{i}}^{t} f(s, x(s)) d s, & t \in\left(s_{i}, t_{i+1}\right], \\ & i=0,1,2,3, \ldots, m .\end{cases}
$$

Assume that $x(t)$ and $\bar{x}(t)$ are the two solutions of (2) satisfying the initial conditions $x\left(t_{0}+0 ; t_{0}, x_{0}\right)=x_{0}$ and $\bar{x}\left(t_{0}+0 ; t_{0}, \bar{x}_{0}\right)=\bar{x}_{0}$, respectively. Now, referring to [14], let us define the stability of solutions in the sense of Lyapunov.

Definition 2 (see [14]). The solution $\bar{x}(t)=x\left(t, t_{0}, \bar{x}_{0}\right)$ of (2) is said to be stable, if, for each $\epsilon>0, \exists$ a $\delta=\delta\left(t_{0}, \epsilon\right)>0$ such that, for any solution $x(t)=x\left(t, t_{0}, x_{0}\right)$ of (2), the inequality $\left\|x_{0}-\bar{x}_{0}\right\| \leq \delta \Rightarrow\|x(t)-\bar{x}(t)\|<\epsilon$, for all $t \geq t_{0}$.

Definition 3 (see [14]). The solution $\bar{x}(t)=x\left(t, t_{0}, \bar{x}_{0}\right)$ of (2) is said to be uniformly stable, if, for each $\epsilon>0, \exists \mathrm{a}$ $\delta=\delta(\epsilon)>0$ such that, for any solution $x(t)=x\left(t, t_{0}, x_{0}\right)$ of (2), the inequality $\left\|x_{0}-\bar{x}_{0}\right\| \leq \delta \Rightarrow\|x(t)-\bar{x}(t)\|<\epsilon$, for all $t \geq t_{0}$.

Definition 4 (see [14]). The solution $\bar{x}(t)=x\left(t, t_{0}, \bar{x}_{0}\right)$ of (2) is said to be uniformly asymptotically stable, if, for each $\epsilon>0$, $\exists$ a $\delta=\delta(\epsilon)>0$ and a $\Gamma=\Gamma(\epsilon)>0$ such that, for any solution $x(t)=x\left(t, t_{0}, x_{0}\right)$ of $(2)$, the inequality $\left\|x_{0}-\bar{x}_{0}\right\| \leq$ $\delta \Rightarrow\|x(t)-\bar{x}(t)\|<\epsilon$, for all $t \geq t_{0}+\Gamma$.

Let us introduce the sets

$$
\begin{array}{r}
G_{i}=\left\{(t, x, \bar{x}) \in[0, T] \times R \times R: t \in\left(s_{i}, t_{i+1}\right]\right\} \\
i=0,1,2,3, \ldots, m \text { with } G=\bigcup_{i=0}^{T} G_{i}, \\
H_{i}=\left\{(t, x, \bar{x}) \in[0, T] \times R \times R: t \in\left(t_{i}, s_{i}\right]\right\} \\
i=0,1,2,3, \ldots, m \text { with } H=\bigcup_{i=0}^{T} H_{i} .
\end{array}
$$

Definition 5. A function $V:[0, T] \times R \times R \rightarrow R_{+}$is said to belong to class $V_{0}$ if

(i) $V$ is continuous in $G_{i}, i=0,1,2, \ldots, m$;

(ii) $V$ is locally Lipschitz continuous in its second and third argument on each of $G_{i}, i=0,1,2, \ldots, m$;

(iii) $V(t, \bar{x}, \bar{x})=0$;

(iv) $V\left(t+0, g_{i}(t, x), g_{i}(t, \bar{x})\right) \leq V(t, x, \bar{x})$ for each $(t, x, \bar{x}) \in H_{i}, i=0,1,2, \ldots, m$;

(v) for $(t, x, \bar{x}) \in G_{i}$, one defines $D^{+} V(t, x, \bar{x})=$ $\operatorname{Lim}_{h \rightarrow 0} \operatorname{Sup}(1 / h)[V(t+h, x+h f(t, x), \bar{x}+h \bar{x} f(t, \bar{x}))-$ $V(t, x, \bar{x})]$;

(vi) for $s_{i}$ in $t_{i}<s_{i} \leq t_{i+1}$ in system (2), $V\left(s_{i}-0, x, \bar{x}\right)=$ $V\left(s_{i}, x, \bar{x}\right)$ and $V\left(s_{i}+0, x, \bar{x}\right)=\operatorname{Lim}_{t \rightarrow s_{i}+0} V\left(s_{i}, x, \bar{x}\right)$.

Note that if $x(t)$ is a solution of system (2), then $D_{(2)}^{+} V(t, x, \bar{x})=V_{(2)}^{\prime}(t, x, \bar{x})$.

We will now use the following class of functions:

$$
\begin{aligned}
& K=\left\{a \in C\left[R_{+}, R_{+}\right]:\right. \\
& \quada(r) \text { is strictly increasing and } a(0)=0\} .
\end{aligned}
$$

\section{Theoretical Results}

Concerning the solution of system (3), referring to [10, 12], we introduce the following assumptions:

(H1) $f \in C(J \times R, R)$;

(H2) there exists a positive constant $L_{f}$ such that $\| f\left(t, x_{1}\right)-$ $f\left(t, x_{2}\right)\left\|\leq L_{f}\right\| x_{1}-x_{2} \|$ for each $t \in[0, T]$ and all $x_{1}$, $x_{2} \in R$; 
(H3) there exists a positive constant $L_{g_{i}}, i=1,2,3, \ldots, m$, such that $\left\|g_{i}\left(t, x_{1}\right)-g_{i}\left(t, x_{2}\right)\right\| \leq L_{g_{i}}\left\|x_{1}-x_{2}\right\|$ for each $t \in\left[t_{i}, s_{i}\right]$ and all $x_{1}, x_{2} \in R$. Also $\max \left\{L_{g_{i}}\right\}=L$ for $i=1,2,3, \ldots, m$;

(H4) $f: J \times R \rightarrow R$ is strongly measurable for the first variable and is continuous for the second variable. There exists a positive constant $L_{f}^{\prime}$ and a nondecreasing function $W_{f} \in C\left([0, \infty), R_{+}\right)$such that $|f(t, x)| \leq L_{f}^{\prime} W_{f}(x)$ for each $t \in J$ and all $x \in R$.

The following is the result regarding the existence of unique solutions of system (3).

Theorem 6. Assume that (H1), (H2), and (H3) are satisfied. Then, problem (3) has unique solution $x \in P C^{1}(J, R)$ provided that

$$
\max \left\{L_{g_{i}}+L_{f}\left(t_{i+1}-s_{i}\right), L_{f} t_{1}: i=1,2,3, \ldots, m\right\}<1 .
$$

Proof. Let $\Lambda: P C^{1}(J, R) \rightarrow P C^{1}(J, R)$ be defined by $\Lambda(x(0))=x_{0}, \Lambda(x(t))=g_{i}(t, x(t))$ for $t \in\left(t_{i}, s_{i}\right]$ and $\Lambda(x(t))==g_{i}\left(s_{i}, x\left(s_{i}\right)\right)+\int_{s_{i}}^{t} f(s, x(s)) d s, t \in\left[s_{i}, t_{i+1}\right]$.

From the assumption, it is clear that $\Lambda$ is well defined.

Moreover, for $x, y \in P C^{1}(J, R), i=1,2,3, \ldots, m$, and $t \in$ $\left[s_{i}, t_{i+1}\right]$, we get

$$
\begin{aligned}
\|\Lambda(x(t))-\Lambda(y(t))\|= & \| g_{i}\left(s_{i}, x\left(s_{i}\right)\right)+\int_{s_{i}}^{t} f(s, x(s)) d s \\
& -g_{i}\left(s_{i}, y\left(s_{i}\right)\right)-\int_{s_{i}}^{t} f(s, y(s)) d s \| \\
\leq & \left\|g_{i}\left(s_{i}, x\left(s_{i}\right)\right)-g_{i}\left(s_{i}, y\left(s_{i}\right)\right)\right\| \\
& +\| \int_{s_{i}}^{t} f(s, x(s)) d s \\
\leq & L_{g_{i}}\|x-y\|_{P C^{1}(J, R)} \\
& +\int_{s_{i}}^{t}\|f(s, x(s))-f(s, y(s))\| d s \\
\leq & L_{g_{i}}\|x-y\|_{P C^{1}(J, R)} f(s, y(s)) d s \| \\
& +\int_{s_{i}}^{t} L_{f}\|x(s)-y(s)\| d s \\
\leq & L_{g_{i}}\|x-y\|_{P C^{1}(J, R)} \\
& +L_{f}\|x-y\|_{P C^{1}(J, R)}\left(t_{i+1}-s_{i}\right) \\
= & \left\{L_{g_{i}}+L_{f}\left(t_{i+1}-s_{i}\right)\right\}\|x-y\|_{P C^{1}(J, R)} \\
& \\
& \\
& \\
&
\end{aligned}
$$

and hence $\|\Lambda(x(t))-\Lambda(y(t))\|_{C\left(\left[s_{i}, t_{i+1}\right], R\right)} \leq\left\{L_{g_{i}}+L_{f}\left(t_{i+1}-\right.\right.$ $\left.\left.s_{i}\right)\right\}\|x-y\|_{P C^{1}(J, R)}$.
Similarly, we obtain

for $t \in\left[t_{i}, s_{i}\right]$,

$$
\|\Lambda(x(t))-\Lambda(y(t))\|_{C\left(\left[t_{i}, s_{i}\right], R\right)} \leq L_{g_{i}}\|x-y\|_{P C^{1}(J, R)},
$$

for $t \in\left[0, t_{1}\right]$,

$$
\|\Lambda(x(t))-\Lambda(y(t))\|_{C\left(\left[t_{i}, s_{i}\right], R\right)} \leq L_{f} t_{1}\|x-y\|_{P C^{1}(J, R)} .
$$

Thus, from the hypothesis $(*)$ in the statement, we see that $\|\Lambda(x)-\Lambda(y)\|_{P C^{1}(J, R)} \leq\|x-y\|_{P C^{1}(J, R)}$ which implies that $\Lambda(\cdot)$ is a contraction map and problem (3) has unique solution in $P C^{1}(J, R)$.

Concerning the existence results of the solutions, the following result is stated without proof (referred to in [12] and proved in [10]).

Theorem 7. Assume that (H3) and (H4) are satisfied and the functions $g_{i}(\cdot, 0)$ are bounded. Then, problem (3) has at least one solution $x \in P C^{1}(J, R)$ provided that

$$
\begin{aligned}
\operatorname{Lim} \sup _{r \rightarrow \infty} \frac{W_{f}(r)}{r} \max \left\{2 L_{g_{i}}+L_{f}^{\prime}\left(t_{i+1}-s_{i}\right),\right. \\
\left.L_{f}^{\prime} t_{1}: i=1,2,3, \ldots, m\right\}<1 .
\end{aligned}
$$

Now, we establish the result for uniform stability of solutions of (2) by employing piecewise Lyapunov functions.

Theorem 8. Assume that (H1), (H2), and (H3) are satisfied. Let there exist functions $V \in V_{0}$ and $a, b \in K$ such that

$$
\begin{array}{r}
a(\|x-\bar{x}\|) \leq V(t, x, \bar{x}) \leq b(\|x-\bar{x}\|), \\
t \in[0, T], \quad x, \bar{x} \in R, \\
V_{(2)}^{\prime}(t, x, \bar{x}) \leq 0, \quad(t, x, \bar{x}) \in G_{i} \\
\text { for each } i=1,2,3, \ldots, m .
\end{array}
$$

Then, the solution $\bar{x}(t)$ of system (2) is uniformly stable.

Proof. Let $\epsilon>0$ be chosen. Choose $\delta=\delta(\epsilon)>0$ so that $b(\delta)<a\left(\epsilon /\left(1-L_{q}\right)\right)$. Let $t_{0} \in[0, T], x_{0}, \bar{x}_{0} \in R$, with $\| x_{0}-$ $\bar{x}_{0} \|<\delta$ and let $x(t)=x\left(t, t_{0}, x_{0}\right), \bar{x}(t)=x\left(t, t_{0}, \bar{x}_{0}\right)$ be the solution of (2).

When $(t, x, \bar{x}) \in G_{i}$, that is, $t \in\left(s_{i}, t_{i+1}\right]$, from the properties of the function $V$ and conditions (10) and (11), we get

$$
\begin{aligned}
a \| & x\left(t, t_{0}, x_{0}\right)-\bar{x}\left(t, t_{0}, \bar{x}_{0}\right) \| \\
& \leq V\left(t, x\left(t, t_{0}, x_{0}\right), \bar{x}\left(t, t_{0}, \bar{x}_{0}\right)\right) \leq V\left(t_{0}+0, x_{0}, \bar{x}_{0}\right) \\
& \leq b\left\|x_{0}-\bar{x}_{0}\right\|<b(\delta)<a(\epsilon) \\
\Longrightarrow & \left\|x\left(t, t_{0}, x_{0}\right)-\bar{x}\left(t, t_{0}, \bar{x}_{0}\right)\right\|<\epsilon ; \quad t \in\left(s_{i}, t_{i+1}\right] .
\end{aligned}
$$


When $(t, x, \bar{x}) \in H_{i}$, that is, $t \in\left(t_{i}, s_{i}\right]$, from (10) and condition (iv) of Definition 5, we get

$$
\begin{aligned}
a & \left\|x\left(t, t_{0}, x_{0}\right)-\bar{x}\left(t, t_{0}, \bar{x}_{0}\right)\right\| \\
& \leq V\left(t, x\left(t, t_{0}, x_{0}\right), \bar{x}\left(t, t_{0}, \bar{x}_{0}\right)\right) \leq V\left(t, g_{i}(t, x), g_{i}(t, \bar{x})\right) \\
& \leq V\left(t_{0}, x_{0}, \bar{x}_{0}\right) \leq b(\delta)<a(\epsilon) \\
\Longrightarrow & \left\|x\left(t, t_{0}, x_{0}\right)-\bar{x}\left(t, t_{0}, \bar{x}_{0}\right)\right\|<\epsilon ; \quad t \in\left(t_{i}, s_{i}\right] .
\end{aligned}
$$

Thus, from inequalities (12) and (13), we find that, for each $\epsilon>0, \exists$ a $\delta=\delta(\epsilon)>0$ such that, for any solution $x(t)=$ $x\left(t, t_{0}, x_{0}\right)$ of (2), the inequality $\left\|x_{0}-\bar{x}_{0}\right\| \leq \delta \Rightarrow \| x(t)-$ $\bar{x}(t) \|<\epsilon$ for $t \geq t_{0}$ and $t \in[0, T]$.

Hence, the solution $\bar{x}(t)$ of system (2) is uniformly stable.

Theorem 9. Assume that all the conditions of Theorem 8 except (11) hold and condition (11) is replaced by the following:

$$
\begin{array}{r}
V_{(2)}^{\prime}(t, x, \bar{x}) \leq-c(\|x-\bar{x}\|), \quad(t, x, \bar{x}) \in G_{i} \\
\text { for each } i=1,2,3, \ldots, m .
\end{array}
$$

Then, the solution $\bar{x}(t)$ of system (2) is uniformly asymptotically stable.

Proof. Let $\epsilon>0$ be given and let the number $\eta=\eta(\epsilon)>0$ be chosen so that $b(\eta)<a(\epsilon)$. Let $\alpha>0$ be a positive constant such that $\alpha<\eta$. Let $\Gamma=\Gamma(\alpha, \epsilon)>0$ be such that $\Gamma>b(\alpha) / c(\eta)$ and $t_{0}+\Gamma \leq T$.

For any $t \in\left[t_{0}, T\right]$, denote $V_{t, \alpha}^{-1}=\{x \in R: V(t+0, x, \bar{x}) \leq$ $a(\alpha)\}$.

Also, from condition (10), for any $x, \bar{x} \in R$,

$$
a(\|x-\bar{x}\|) \leq V(t+0, x, \bar{x}) \leq a(\alpha) \Longrightarrow\|x-\bar{x}\| \leq \alpha .
$$

Therefore, $V_{t, \alpha}^{-1}=\{x \in R:\|x-\bar{x}\| \leq \alpha\}$.

It follows that for any $x_{0} \in V_{t_{0}, \alpha}^{-1}$ we have $x\left(t ; t_{0}, x_{0}\right) \in V_{t, \alpha}^{-1}$.

If possible, assume that, for each $t \in\left[t_{0}, t_{0}+\Gamma\right]$, the inequality

$$
\left\|x\left(t ; t_{0}, x_{0}\right)-t_{0}-\bar{x}\left(t ; t_{0}, \bar{x}_{0}\right)\right\| \geq \eta
$$

is valid.

Case 1. If $t \in\left[t_{0}, t_{0}+\Gamma\right]$ such that $(t, x, \bar{x}) \in H_{i}$, then, from (16), we have

$$
\begin{aligned}
& \left\|x\left(t ; t_{0}, x_{0}\right)-t_{0}-\bar{x}\left(t ; t_{0}, \bar{x}_{0}\right)\right\| \geq \eta \\
& \quad \Longrightarrow b(\eta) \leq b\left(\left\|x\left(t ; t_{0}, x_{0}\right)-t_{0}-\bar{x}\left(t ; t_{0}, \bar{x}_{0}\right)\right\|\right) \leq b(\alpha) \\
& \quad \Longrightarrow \eta \leq \alpha,
\end{aligned}
$$

which is a contradiction to the assumption $\alpha<\eta$ and hence (16) is not valid.

Case 2. If $t \in\left[t_{0}, t_{0}+\Gamma\right]$ such that $(t, x, \bar{x}) \in G_{i}$, then, from (14) and (16), we have

$$
\begin{aligned}
& V_{(2)}^{\prime}(t, x, \bar{x}) \leq-c(\|x-\bar{x}\|), \quad(t, x, \bar{x}) \in G_{i} \\
& \Longrightarrow V\left(t_{0}+\Gamma, x\left(t_{0}+\Gamma ; t_{0}, x_{0}\right), \bar{x}\left(t_{0}+\Gamma ; t_{0}, \bar{x}_{0}\right)\right) \\
& \quad-V\left(t_{0}+0, x_{0}, \bar{x}_{0}\right) \\
& \leq-\int_{t_{0}}^{t_{0}+\Gamma} c\left(\left\|x\left(s ; t_{0}, x_{0}\right)-\bar{x}\left(s ; t_{0}, \bar{x}_{0}\right)\right\|\right) d s \\
& \Longrightarrow V\left(t_{0}+\Gamma, x\left(t_{0}+\Gamma ; t_{0}, x_{0}\right), \bar{x}\left(t_{0}+\Gamma ; t_{0}, \bar{x}_{0}\right)\right) \\
& \leq V\left(t_{0}+0, x_{0}, \bar{x}_{0}\right)-\int_{t_{0}}^{t_{0}+\Gamma} c(\eta) d s \\
& \leq b(\alpha)-\Gamma c(\eta)<0,
\end{aligned}
$$

which is a contradiction to the assumption $\Gamma>b(\alpha) / c(\eta)$ and hence (16) is not valid.

Thus, we see that in both cases whether $(t, x, \bar{x}) \in G_{i}$ or $(t, x, \bar{x}) \in H_{i} \exists t^{*} \in\left[t_{0}, t_{0}+\Gamma\right]$ such that $\| x\left(t ; t_{0}, x_{0}\right)-t_{0}-$ $\bar{x}\left(t ; t_{0}, \bar{x}_{0}\right) \|<\eta$.

Then, for $t^{*} \in\left[t_{0}, t_{0}+\Gamma\right]$ and hence for any $t \geq t_{0}+\Gamma$, we have

$$
\begin{gathered}
a(\|x-\bar{x}\|) \leq V(t ; x(t), \bar{x}(t)) \leq V\left(t^{*} ; x\left(t^{*}\right), \bar{x}\left(t^{*}\right)\right) \\
\leq b\left(\left\|x\left(t^{*} ; t_{0}, x_{0}\right)-\bar{x}\left(t^{*}, t_{0}, \bar{x}_{0}\right)\right\|\right) \\
<b(\eta)<a(\epsilon) \\
\Longrightarrow\left\|x\left(t ; t_{0}, x_{0}\right)-\bar{x}\left(t, t_{0}, \bar{x}_{0}\right)\right\|<\epsilon \\
\text { for } t \geq t_{0}+\Gamma .
\end{gathered}
$$

Thus, the solution $\bar{x}(t)$ of system (2) is uniformly asymptotically stable.

\section{Example}

Let $J=[0,2]$ and $0=t_{0}=s_{0}<t_{1}=1<s_{2}=2$. Denote $f(t, x(t))=1 /(1+|x(t)|)$ for $t \in(0,1]$ and $g_{1}(t, x(t))=$ $|x(t)| /(2+|x(t)|)$ for $t \in(1,2]$. Also define functions $a, b \in K$ as $a(t)=(1 / 2) t$ and $b(t)=(3 / 2) t$.

Here, $(t, x, \bar{x}) \in G_{1}$; that is, for $t \in(0,1]$,

$$
\begin{aligned}
\left|f\left(t, x_{1}(t)\right)-f\left(t, x_{2}(t)\right)\right| & =\left|\frac{1}{1+\left|x_{1}(t)\right|}-\frac{1}{1+\left|x_{2}(t)\right|}\right| \\
& =\left|\frac{\left|x_{2}(t)\right|-\left|x_{1}(t)\right|}{\left(1+\left|x_{2}(t)\right|\right)\left(1+\left|x_{1}(t)\right|\right)}\right| \\
& \leq|| x_{1}(t)|-| x_{2}(t)|| \\
& \leq\left|x_{1}(t)-x_{2}(t)\right| .
\end{aligned}
$$


Therefore, we have $L_{f}=1$. Similarly, it can be seen that, for $t \in(1,2], L_{g_{1}}=1 / 2$.

Now, consider the system

$$
\begin{aligned}
& x^{\prime}(t)=\frac{1}{1+|x(t)|}, \quad t \in(0,1], \\
& x(t)=\frac{|x(t)|}{2+|x(t)|}, \quad t \in[1,2) .
\end{aligned}
$$

Let $V(t, x, \bar{x})=\|x(t)-\bar{x}(t)\|$. Without loss of generality, we assume that $\|x(t)\| \geq\|\bar{x}(t)\|$.

Clearly, $V(t, \bar{x}, \bar{x})=0$. Also $V$ is continuous and locally Lipschitz in second and third argument on $G_{1}$.

Also, for $(t, x, \bar{x}) \in G_{1}$,

$$
\begin{aligned}
V_{(2)}^{\prime}(t, x, \bar{x}) & =\left[x^{\prime}-\bar{x}^{\prime}\right] \operatorname{Sign}(x-\bar{x}) \\
& =\left\{\frac{1}{[1+|x(t)|]}-\frac{1}{[1+|\bar{x}(t)|]}\right\} \\
& =\left\{\frac{|\bar{x}(t)|-|x(t)|}{[1+|x(t)|][1+|\bar{x}(t)|]}\right\} \leq 0 .
\end{aligned}
$$

For $(t, x, \bar{x}) \in H_{1}$, that is, for $t \in(1,2]$,

$$
\begin{aligned}
V( & \left.+0, g_{i}(t, x), g_{i}(t, \bar{x}) \in H_{i}\right) \\
& =V\left(t+0, \frac{|x(t)|}{2+|x(t)|}, \frac{|\bar{x}(t)|}{2+|\bar{x}(t)|}\right) \\
& =\left\|\frac{|x(t)|}{2+|x(t)|}-\frac{|\bar{x}(t)|}{2+|\bar{x}(t)|}\right\| \\
& =\left\|\frac{2|x(t)|-2|\bar{x}(t)|}{[2+|x(t)|][2+|\bar{x}(t)|]}\right\| \leq \frac{2}{4}\||x(t)|-|\bar{x}(t)|\| \\
& \leq \frac{1}{2}\||x(t)|-|\bar{x}(t)|\| \leq V(t, x, \bar{x}) .
\end{aligned}
$$

Also, $a(\|x-\bar{x}\|)=(1 / 2)\|x-\bar{x}\| \leq\|x-\bar{x}\|=V(t, x, \bar{x}) \leq$ $(3 / 2)\|x-\bar{x}\|=b(\|x-\bar{x}\|)$.

Thus, we see that all the conditions of Theorem 8 are satisfied and hence system (21) is uniformly stable.

\section{Conclusion}

Stability of solutions of impulsive ordinary differential equations with instantaneous impulses has been discussed extensively in the past [1-9]. Motivated by the recent work [10-13], in this paper, a new class of ordinary differential equations with noninstantaneous impulses has been studied and uniform stability and uniform asymptotic stability of solutions of such systems are investigated by using piecewise Lyapunov functions.

\section{Conflict of Interests}

The authors declare that there is no conflict of interests regarding the publication of this paper.

\section{References}

[1] Y. Zhang and J. Sun, "Eventual stability of impulsive differential systems," Acta Mathematica Scientia, Series B, vol. 27, no. 2, pp. 373-380, 2007.

[2] V. Lakshmikantham, D. D. Bainov, and P. S. Simeonov, Theory of Impulsive Differential Equations, vol. 6 of Series in Modern Applied Mathematics, World Scientific, River Edge, NJ, USA, 1989.

[3] S. Ahmad and I. Stamova, "Stability criteria for impulsive Kolmogorov-type systems of nonautonomous differential equations," Rendiconti dell' Istituto di matematica dell' Università di Trieste, vol. 44, pp. 19-32, 2012.

[4] R. I. Gladilina and A. O. Ignat'ev, "On retention of impulsive system stability under perturbations," Automation and Remote Control, vol. 68, no. 8, pp. 1364-1371, 2007.

[5] V. Raghavendra and M. Rama Mohana Rao, "Differential systems with impulsive perturbations and an extension of Lyapunov's method," Indian Journal of Pure and Applied Mathematics, vol. 13, no. 8, pp. 890-897, 1982.

[6] G. K. Kulev and D. D. Bainov, "Asymptotic stability of systems with impulses by the direct method of Lyapunov," Bulletin of the Australian Mathematical Society, vol. 38, no. 1, pp. 113-123, 1988.

[7] N. A. Perestyuk, V. A. Plotnikov, A. M. Samoilenko, and N. V. Skripnik, Differntial Equations with Impulsive Effects: Multi Valued Right Hand Sides with Discontinuities, Walter De Gruyter, GmbH \& Co. KG, Berlin, Germany, 2011.

[8] M. Benchohra, J. Henderson, and S. Ntouyas, Impulsive Differential Equations and Inclusions, Hindawi Publishing Corporation, New York, NY, USA, 2006.

[9] A. Sood and S. K. Srivastava, " $\Psi$-eventual stability of differential systems with impulses," Global Journal of Science Frontier Research: Mathematics \& Decision Sciences, vol. 14, no. 6, pp. 1-8, 2014.

[10] E. Hernandez and D. O’Regan, "On a new class of abstract impulsive differential equations," Proceedings of the American Mathematical Society, vol. 141, no. 5, pp. 1641-1649, 2013.

[11] M. Pierri, D. O’Regan, and V. Rolnik, "Existence of solutions for semi-linear abstract differential equations with not instantaneous impulses," Applied Mathematics and Computation, vol. 219, no. 12, pp. 6743-6749, 2013.

[12] Y. Liao and J. Wang, "A note on stability of impulsive differential equations," Boundary Value Problems, vol. 2014, article 67, 2014.

[13] X. Li and J. Wang, "Ulam Hayer-Rassias stability of semi linear differential equations with impulses," Electronic Journal of Differential Equations, vol. 2013, no. 172, pp. 1-8, 2013.

[14] S. Ahmad and M. R. M. Rao, Theory of Differential Equations, Affiliated East West Press Private, 1999. 


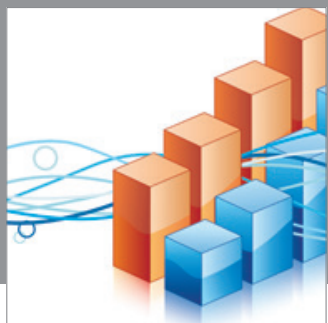

Advances in

Operations Research

mansans

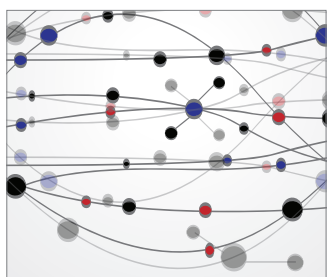

The Scientific World Journal
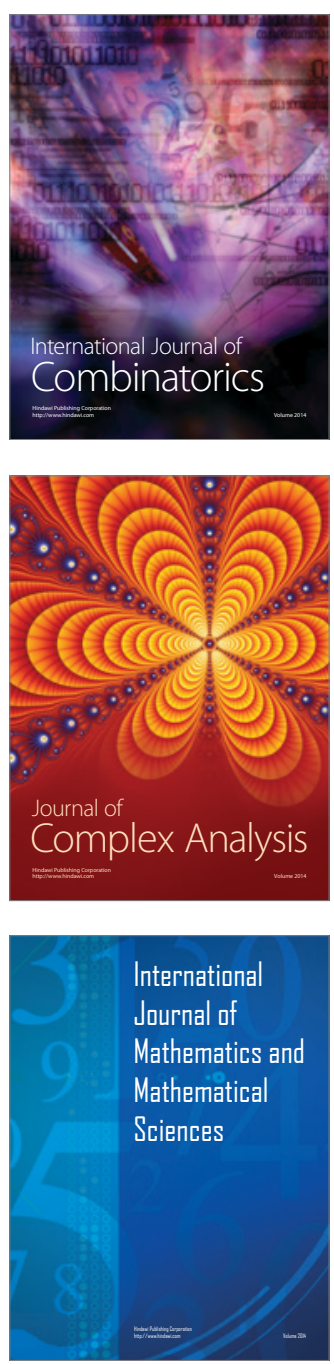
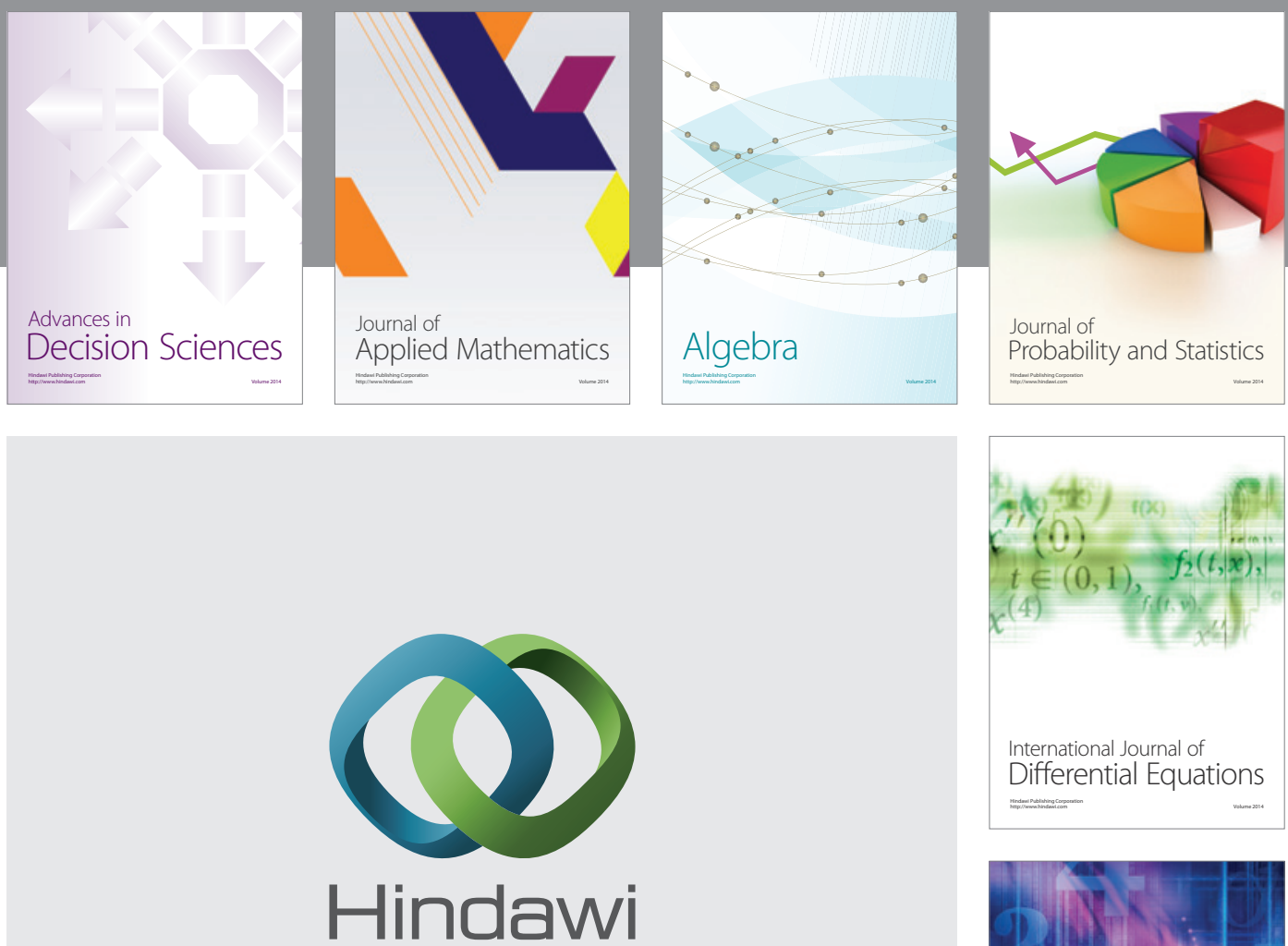

Submit your manuscripts at http://www.hindawi.com
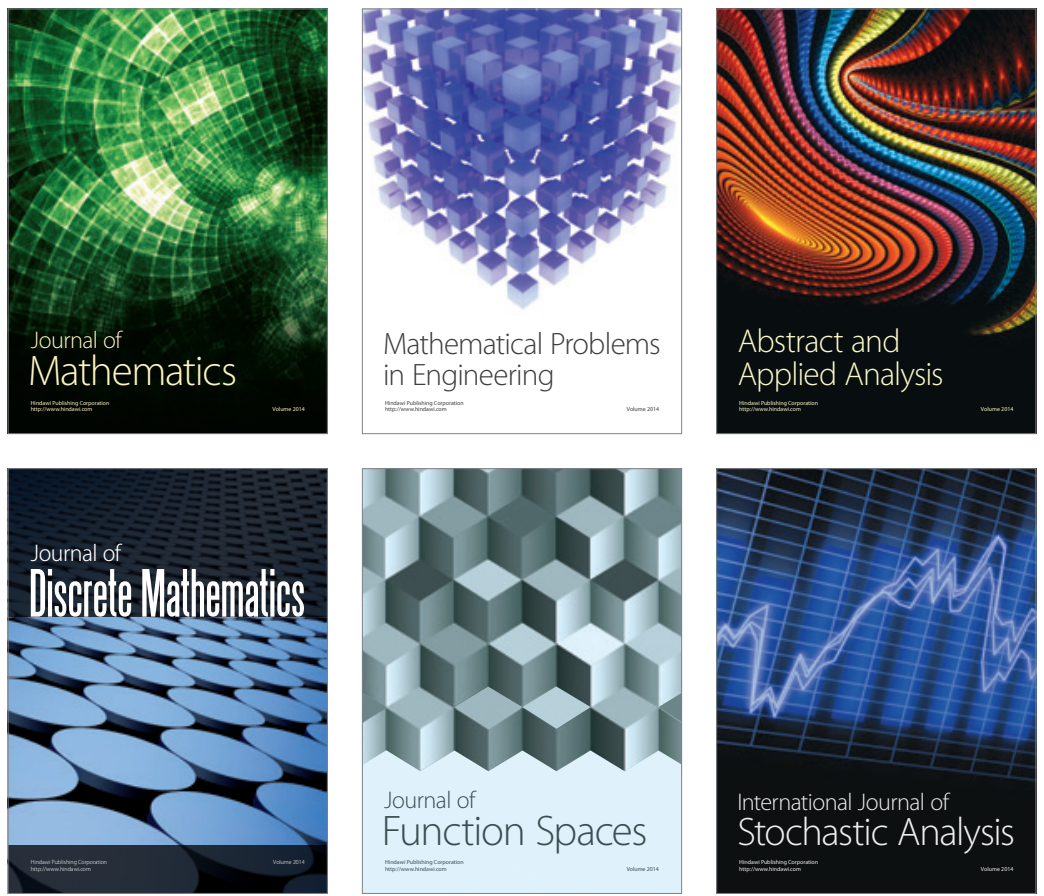

Journal of

Function Spaces

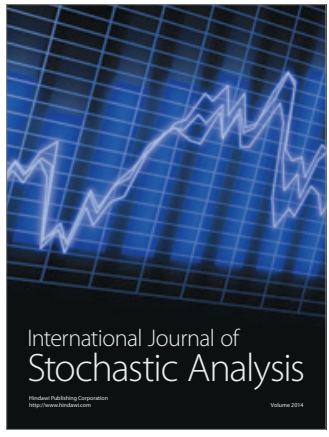

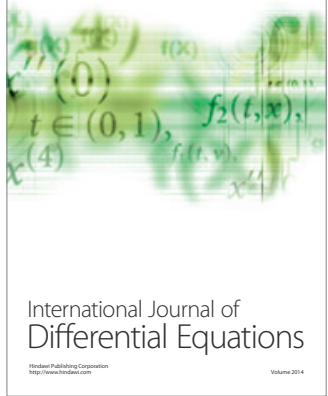
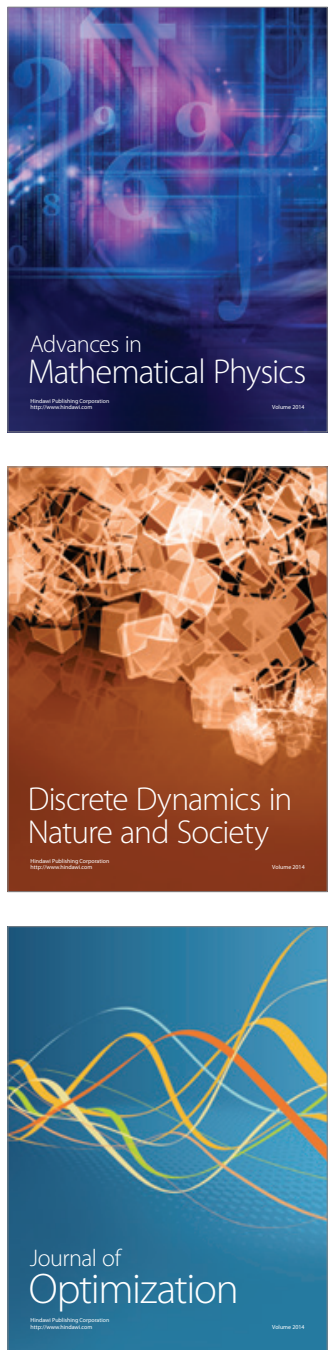